\title{
Working within resource constraints: A qualitative segmentation study.
}

\begin{abstract}
Social marketers are often operating with scarce financial and human resources preventing costly quantitative segmentation methods from being applied. This paper explores the utility of applying qualitative research to identify groups that can be used for social marketing planning and program implementation. Drawing from a single water use case, six focus groups employing a semi-structured discussion guide involving 43 participants who were living and working within the site were conducted. Focus group interviews were audiotaped and manual coding was used to identify major segments within one water use market. Four distinctive segments emerged. The generated segments were denominated comfort users, careless users, price-sensitive users, and contradictory users respectively, with comfort users constituting the largest segment. This paper contributes to the literature by offering a method permitting social marketers operating within limited budgets to apply segmentation. Research limitations and future research directions are outlined.
\end{abstract}

\section{KEYWORDS}

Focus Group, Social Marketing, Qualitative Segmentation, United Arab Emirates. 
2 Fresh water is a scarce resource in many parts of the developing world (Mekonnen \&

\section{Introduction}

Hoekstra, 2016). High temperatures and low precipitation in the Arabian Gulf make it one of the world's harshest environments. In the United Arab Emirates (UAE) for example, the average rainfall varies from $60 \mathrm{~mm}$ to $160 \mathrm{~mm}$ per year (Haque, AlKhaiat, \& John, 2007); only 7 mm of rain fell in 1999-2000 (Murad, Al Nuaimi, \& Al Hammadi, 2007). The country's average per capita consumption of water, at 550 litres a day, is above average water use levels globally (Reporter, 2016; Szabo, 2011). Szabo (2011) found that more than half (57\%) of residential water consumption was attributable to luxury lifestyle habits and little awareness of water scarcity in the UAE. The country's growth in population is expected to lead to an even greater demand for water, estimated to increase by 50\% over the period 2015-2020 (Ghaith \& Abusitta, 2014). Strong economic development will, in addition, increase the demand for water by $40 \%$ over the next decade (Mohamed \& Al-Mualla, 2010).

Two possible solutions to the problem of water scarcity are available to the UAE. These are: 1) increasing supply, i.e. developing new sources of water, such as by recycling wastewater, or continuing to build costly, energy-consuming and salinity-increasing seawater desalination facilities; and 2) reducing demand, i.e. conserving existing resources through changing water consumption behaviour. A major obstacle to implementing behavioural change solutions to excess water consumption is often imputed to public unwillingness to adopt new, or alternative water consumption behaviours (Longfield et al., 2016).

Recently, social marketing has been recognised as a credible behaviour change discipline and has been widely adopted to foster social change (Rundle-Thiele, Kubacki, Tkaczynski, \& Parkinson, 2015). Various social issues have been tackled by 
social marketing programs in a range of fields that include lifestyle-related health issues (Kamada et al., 2013), condom use (Purdy, 2011), childhood healthy eating (Keihner et al., 2011), alcohol and substance abuse (Glik, Prelip, Myerson, \& Eilers, 2008) and diet and physical activity (Huhman, Bauman, \& Bowles, 2008) as well as transport and environmental concerns (Lefebvre, 2011). Water consumption behaviour has similarly been previously targeted by social marketing interventions (Kubacki, Rundle-Thiele, Pang, \& Buyucek, 2015). For example, a social marketing program in Florida, U.S. directed at water consumption behaviour was able, through the use of positive messaging, to convince consumers of their role in the solution to the problem of overusing the resource Jesperson (2005). In South East Australia, research by Lowe, Lynch, and Lowe (2014) demonstrated the success of a social marketing campaign that aimed to reduce water consumption to an enduring, sustainable level. While there are precedents of successfully targeting excess water consumption using social marketing in the United States and Australia, there is scant research concerning the UAE water use market.

To heighten the visibility of social marketing programs, program developers must deliver programs that more directly reflect the different needs and wants of identified groups within a target audience (Andreasen, 2012). Literature indicates that segmentation is rarely applied by social marketers (Kubacki \& Rundle-Thiele, 2017), and this may be a direct result of a lack of capacity in the sector, given that complex multivariate procedures requiring large sample sizes dominate segmentation practice (Dietrich, Rundle-Thiele, \& Kubacki, 2017).

\section{Market segmentation}

Market segmentation is the process of clustering groups of individuals or objects with similar characteristics, needs, wants, and behaviours into a homogeneous cluster or 
group (Dibb, 2017). In social marketing, segmentation refers to developing a strong and meaningful understanding of groups within a population that share common characteristics and behaviours as the basis of developing a suitably tailored social marketing project that is segment-specific (Dolnicar, Grün, \& Leisch, 2016; Donovan \& Henley, 2003; French, 2017). As indicated by French and Gordon (2015), social marketers can add real value to programs by applying segmentation. However, despite the reported benefits of market segmentation, segmentation methods are underrepresented in social marketing programs. For example, of 93 interventions assessed in a recent review, a total of only $15(16 \%)$ reported applying segmentation in social marketing programs (Dietrich et al., 2017).

Conducting consumer research is essentially for individual orientation (Andreasen, 2002). Social marketing research, therefore, requires a comprehensive understanding of a target audience's aspirations, needs, values, and their everyday lives and behaviours (Andreasen, 2012). Furthermore, market segmentation research offers unique insight into consumers' perceptions of benefits, costs and products, as well as illuminating other factors such as perceived threat, self-efficacy, and social influences that might motivate, or discourage, the target audience from adopting more desirable behaviours (Andreasen, 2012). Market segmentation also produces information about distinct population subgroups and the social and cultural environments in which consumers make decisions relative to certain behaviours. The information generated from market segmentation research is an important consideration in strategic marketing decision-making relevant to the selection of segments to be targeted, and the benefits and the costs of the program (French, 2017). The types of data that can be utilised to construct a segmentation model vary in scope and insight. Segmentation data can be derived from a range of segmentation 
models and methodologies (French, 2017). There are, for example, a variety of quantitative approaches to segmentation. Data-driven segmentation methods for segmenting a target audience include two-step cluster analysis (Rundle-Thiele et al., 2015), K-means algorithm (Jain, 2010), and data mining (Berkhin, 2006). The social marketing segmentation process should be based on scientific research to identify the different segments of the target audience. However, this process need not be expensive or complex (Grier \& Bryant, 2005).

While the benefits of employing quantitative techniques in segmentation are acknowledged, their processes may present some concerns for some social marketing programs. First, quantitative segmentation processes cannot be applied in markets with small numbers; current best practice guidelines suggest 100 cases are needed for each variable measured (Dolnicar et al., 2016). Second, many social marketing projects lack sufficient funds for inclusion of sophisticated quantitative segmentation studies, which require time, expertise and, therefore, sufficient finance for the activity. Third, quantitative segmentation often faces a shortage of high-level expertise necessary to conduct an advanced successful segmentation study (Randle \& Dolnicar, 2017). A qualitative segmentation approach is considered an alternative approach to segmenting the market when social marketers have limited access to the target audience (Lefebvre, 2013), and where populations of interest are small (Randle \& Dolnicar, 2015).

\section{Qualitative segmentation approach}

According to Dolnicar et al. (2016), there is not a single best approach to social marketing segmentation. The most applicable market segmentation approach in any given situation depends on at least two factors: (1) the strategic marketing goals of the 
segmentation that will inform program design; and (2) the quality of the data required as the basis for the segmentation study.

Qualitative segmentation is a process of using qualitative methods, approaches, and techniques to group and cluster similarly targeted audience members according to their demographic, geographic, psychographic, and behavioural characteristics (de Visser et al., 2015). A qualitative market segmentation approach can generate unique and rich insights into marketing phenomena, and reduce the cost of conducting segmentation studies compared to data-driven segmentation approaches (Bond \& Morris, 2003). Qualitative segmentation is important in identifying and managing the target market because of its efficient use of resources. Key strengths of a qualitative approach are its ability to generate situational insights, such as pinpointing the major competitor of the behaviour change and ascertaining the extent of need in the segment, assess the segment of interest's responsiveness to change, and discover what social marketers offer in exchange (Atkinson \& Hammersley, 1994; Carson, Gilmore, Perry, \& Gronhaug, 2001; Lefebvre, 2013). In one example, using qualitative segmentation methods, namely observation and interviews, the San Diegobased social marketing behaviour change agency RESCUE segmented teenagers based on their risk behaviour (Dietrich et al., 2017). These qualitative approaches revealed distinct segments of the teenage audience (Dietrich et al., 2017).

In the commercial marketing domain, qualitative methods have been successfully used to generate segments, and to gain market insights. A study conducted by Charmaz (2006), for example, used structured in-depth interviews and semi-structured questions about participants' brand loyalty to identify distinct segments. Following that study, a semi-structured interview protocol was used to collect qualitative data from brand professionals by asking them a series of open- 
ended questions about their career, job profile, brand management experience, managerial level and role (Grougiou \& Pettigrew, 2009). Recently, a segmentation study from Turkey segmented the container shipping service market. A total of 20 semi-structured interviews were conducted with managers to identify and understand the characteristics of four distinct segments (Balci \& Cetin, 2017).

\section{Method}

The University of Sharjah (UoS) is one of the largest higher educational institutions in the UAE, with more than 30,000 students, staff, and faculty members. Its 280,000 square metres of building area includes student dorms, and faculty and staff housing units. Across the green landscape of the university's main campus, there are football fields, large parks, and huge fountains featuring constantly running. Water is freely available across all campus facilities.

In this study, knowledge/awareness, beliefs, habits, and facilitating factors that might influence participants' water consumption behaviours were explored in order to identify whether different segments could be discerned. This study employed a single case study design (Yin, 2012) in which the case analysed was the UoS water use market. Six focus groups were formulated of people living, studying, and working inside the campus, consisting of (1) female students, (2) male students, (3) graduate students, (4) faculty members, (5) staff members, and (6) a mixture of staff, faculty, and students. Qualitative segmentation involves applying qualitative data collection and analysis methods, approaches, and techniques to group or 'cluster' respondents according to their opinions, attitudes, behaviours, beliefs, emotions, and habits (de Visser et al., 2015). Qualitative segmentation can include experiences, obstacles, personal impressions, feelings, and opinions of the UoS residents (Hofmeister-Tóth, Kelemen, \& Piskóti, 2011). 


\section{Focus groups sessions}

151 In this project the focus group technique was selected as the most appropriate method

152 for gathering the data for the qualitative segmentation. Focus group technique enabled

153

154

155

the researcher to get closer to the subject's perspective in order to drive the required data needed for segmentation process, through relatively unstructured and fairly freeflowing interview with a small group of people to explore their awareness/knowledge, attitude, emotions, facilitating factors, and their previous indoor, outdoor, and personal hygiene habits. (Stokes \& Bergin, 2006; Zikmund, McLeod, \& Gilbert, 2003). The focus group method enabled the researcher to modify the emphasis and the orientation of the questions during the session with the emergence of new ideas and themes. This was an ideal way to discover variables not previously mentioned in the literature and to add new features to the study.

The focus group technique helped the researcher to obtain insights into informants' interests and solicit their opinions about water consumption behaviour (Berger, Bonime, Goldberg, \& White, 2001; Garee \& Schori, 1996). The main advantages of using the focus group method for this study besides their being inexpensive to run were their relatively speed and ease of conduct (Zikmund et al., 2003).

The purpose of the focus group discussions was to ask a series of open-ended questions, and encourage participants to explore the subjects of importance to them in their own vocabulary, generate their own questions, and pursue their own priorities (Esposito, 2001). Previous social marketing studies aimed at examining water consumption have used the focus group method. Lowe et al. (2014) conducted four focus groups in South East Australia, to understand residents' water consumption habits. 
176 participants were asked 11 open-ended questions. In the first, introductory, stage participants were asked about their knowledge/awareness concerning the water situation and resources in the UAE. The second stage was the exploratory stage in which participants were asked about their actual water consumption behaviours inside and outside their housing. This stage contributed to identifying participants' water-use behaviours. In the third stage, participants were asked about the facilitators that might influence reductions in their water consumption.

The lead researcher acted as a facilitator, or moderator, of the discussion.

Group sessions lasted from 45 to 60 minutes, with an average length of 50 minutes.

The focus group sessions were recorded with the consent of participants and the recordings were later transcribed for analysis.

\section{Sampling Method}

This research used a combination of purposive and convenience sampling techniques

to locate key informants. The use of purposive sampling approach allows for identifying, detecting, and selection of information-rich cases related to the phenomenon of interest (Creswell, 2012).

\section{Sample Size}

The sample size in qualitative research is a matter of judgment and experience of the researcher in evaluating the quality of the data collected, sampling strategy employed, the particular research method, and the research product intended (Sandelowski, 1995). In Patton (2005) there are no rules restricting sample size in qualitative inquiry. For the purposes of this study, a minimum sample was constructed, and a minimum of one focus group session under each unit of analysis was conducted. 
each focus group was 6-8 participants. In the existing study, a total of 6 focus groups

were conducted and were adequate to gain in-depth understanding of perspectives of

participants about water consumption and where was no new information emerged and data saturations achieved.

204

205

206

207

208

\section{Validity and Reliability}

During, and following each focus group session, the researcher took field notes,

which were transcribed immediately to address any areas of uncertainty, and to ensure that any ambiguities could be clarified with the participant(s) before data analysis.

\section{Data analysis}

Qualitative data analysis was used to classify participants based on their knowledge, age, gender, place of living, paying for water usage, beliefs, and water consumption habits. Following procedures outlined by Bryman (2008) and Guba and Lincoln (1994), the researcher coded and/or indexed the data collected throughout the data analysis process, looking for common patterns to cluster the data accordingly so that common segments could be generated. It is important to note that while the process of analysis was taking place, the researcher contacted interviewees for additional data in two instances: (1) to gain a more detailed response; and (2) where doubt or inconsistencies were perceived.

The data were reviewed concurrently and coded consistently. To extract the major segments based on the content analysis of similarities, a careful and thorough examination of the characteristics of the population (i.e. gender, age, paying/not paying for water, living on- or off-campus, nationality, occupation, education level, and habits) that represented each segment was conducted. 


\section{Results}

224 A total of 43 participants, whose demographic characteristics are summarised below

225 in Table 1, voluntarily joined the focus groups from a diverse population of the UoS.

\section{INSERT TABLE 1 HERE}

The sample comprised of 21 males and 22 females; 25 participants were

students, nine were faculty members, and nine were staff-members. A total of 19 participants were local Emirates citizens, and 24 were expatriates. A total of 23 were living inside university accommodation, and 20 lived off-campus. More than half of the participants (33) were not paying for their water usage, and 20 paid for water. The age range of participants was 18-56 years old.

\section{Segmentation bases}

Segmentation bases are the sets of variables used to create homogeneous groups or segments within the heterogeneous target audience (Wedel \& Kamakura, 2012). The individuals who make up a segment necessarily have similar needs, behaviours, and factors that influence their behaviour (Dibb, 2017). This study used the four segmentation bases of demography (gender, age, nationality, role, and education); geography (living inside or outside the campus), psychography (knowledge/awareness, paying for water, and beliefs); and the behavioural dimension of segmentation (water consumption behaviour). Social marketing scholars and practitioners have argued that by relying on only one segmentation base, true market segments are unlikely to be identified (Dibb \& Carrigan, 2013).

This study was able to identify four major segments that best fitted the UoS sample of residents. These four segments were defined as: comfort, careless, contradictory, and price-sensitive users. Table 2 summarises the segmentation bases and variables used to compile segment profiles. 
251 In the profile analysis stage of segmentation the aim is to explore and construct the

252 segments which, in turn, can form the basis of successful marketing strategies

253 (French, 2017). Profiling was used to establish a clear understanding of the segments'

254 similarities and differences, as recommended by Weinstein and Cahill (2014).

255 Furthermore, the new generated segments were designated by a meaningful and

256 interpretive name indicative of the salient information and insights pertinent to each

257 segment (Lefebvre, 2013). During this stage of analysis the researcher asked

258 questions of participants to draw out their concerns, facilitating factors, interests, and

259 beliefs in order to profile each segment (Dietrich et al., 2017). Once insights were

260 gained, a systematic process of analysis was undertaken to consider segment

261 similarities and differences (Longfield et al., 2016). Each identified segment is now

262 described.

263 Comfort users

264 The researchers categorised 16 participants (37\% of focus group participants) as

265 comfort users; their average age was 28 years. This segment consists of local young

266 Emirati, female students, who do not pay for the water they use. They typically live

267 off-campus and are at their first level of higher education. Data from the focus groups

268 showed that the comfort segment lacks the proper knowledge about the UAE's water

269 situation, believing that the country is rich in natural water resources due to its

270 geographic location (surrounded by the Arabian Gulf and Oman Sea) which, they had

271 concluded, meant that water scarcity was not an issue.

"We are lucky in this country; we have two seas, water is always there for our use, government is ready to supply water for residents" (Female, local student, 19 -years-old, living in a family whose water bill is paid by the government). 
"Yes, UAE has enough water resources for the time being, and we should not be worried. I, my concern is to have water for my personal and family use" (Male, faculty-member, 46-year old expat, living inside the campus).

"The most important thing for me is to see my children and my house are clean, without overconsumption, and this is the university's responsibility to supply enough water" (Male, faculty-member, 53-year-old expat, living on campus).

The general consumption behaviour of this segment was to use available water as needed without attention to the quantities consumed. The major concern of this segment was to have running water for their daily requirements. Comfort users agreed that conserving water is necessary, believing that they can do more to save water voluntarily, without any external pressure to do so. Table 3 profiles the individuals in the comfort users' segment.

\section{INSERT TABLE 3 HERE}

\section{Careless users}

The researchers categorised 11 participants ( $25 \%$ of all participants) as careless users. Participants in this segment had an average age of 28 years. Careless users were mostly young, male, local and expat students who lived on campus, and did not pay for their water usage. Again, people in this segment were unaware that the UAE is under water stress, has limited natural water resources, and that their water consumption was quite excessive. Members of this segment habitually used water without heed to their consumption:

"I don't think I can save water because I don't know how" (Male local student, 22-years-old, living on campus).

"The UAE has a lot of water, and I can use it whenever I need it; why should I care?" (Male expat student, 22-year-old, living on campus).

"I take a shower for two hours; I feel the pleasure of warm water running over my body" (Female local student, 20-years-old, living on campus). 
"I wash my car with a hose once a week" (Male expat faculty-member 49 years old, living with his family on campus).

"I wash my t-shirt by itself at the laundry every day" (Male local student, 21years-old, expat, living on campus).

"I am lazy to turn the water off while I am shaving and brushing my teeth; I am not paying for water, why should I care?" (Male local student, 22-yearold, living on campus).

"I wash my cars using the hose, and I clean the driveway from sand almost every week; my cars and my house should look clean" (Male, 39-year-old, local staff, living off-campus, government pays for water usage).

Some highly educated faculty members exhibited careless water consumption behaviours, such as washing their cars in the driveways of their houses using a hose, and taking long showers. The careless users' segment was clearly distinguished by the excessive consumption habits of its members, together with an unwillingness to make any voluntary changes to their individual water consumption behaviour. They made clear their collective belief that it is the responsibility of a third party to make water continuously available. Table 4 sets out the demographic details of the careless segment.

\section{INSERT TABLE 4 HERE}

\section{Contradictory users}

This segment consisted of six people (14\% of all participants) representing males and females with an average age 34.5 years old. The contradictory segment contained people from diverse backgrounds living on campus. Individuals in this segment did not pay for the water they used, and consistent with all other segments, they had high levels of usage. Members in the contradictory segment were educated, and similarly to the comfort and careless segments, people in this group also believed the UAE to have plentiful water. The key distinguishing feature of this segment was their belief in 
the importance of Islamic teachings as they relate to water consumption. People in this segment voiced support for the role of Islamic institutions as guides to reducing excessive water consumption. The misalignment between participants' actual overconsumption behaviour and their Islamic beliefs was evident:

"Islam is encouraging Muslims to use less water... but I use a lot of water" (Female expat student, 22 years old, living on campus).

"We all knew that our prophet Mohammed Hadith said, 'Don't use too much water even if you are living beside a running river'. However, we are not following that, not even when we make ablution 'Wudu' for praying'” (Male expat faculty-member, 55-year old, living on campus).

"I follow Islam instructions in saving water; however, my children, they don't care" (49-year old expat male, living on campus).

The inconsistency between their professed belief in the tenets of their faith which advises Muslims to use water parsimoniously, and actual water consumption behaviour gave this segment the "contradictory users" label. This segment showed their faith in Islam is strong, which could be used by social marketers to target their water consumption behaviour by focusing on the Islamic injunction to conserve precious resources. Table 5 presents the characteristics of the contradictory users' segment.

\section{INSERT TABLE 5 HERE}

\section{Price-sensitive users}

Some participants indicated clearly that cost-free water is a major driver behind their excess water consumption. The price-sensitive users' segment comprised of ten participants (23\% of all participants), whose characteristics are shown in Table 6 . Members of the price-sensitive segment did not pay for their water usage, yet were willing to change their behaviour and reduce their consumption if there was a price tag attached to their consumption: 
"If we pay for water, I will watch my use, and push my children to do so" (Male faculty-member 48-year-old, living on campus).

"Price tag is necessary for residents to control their consumption" (Male faculty-member 55-year-old, living on campus.

\section{INSERT TABLE 6 HERE}

The price-sensitive segment was unique in that they clearly expressed that they would not change their behaviour voluntarily, and would only likely respond to coercive measures, such as a price signal. Social marketers can target this segment by using structured approaches to reducing water consumption via pricing, and more legal water restrictions. which would support moving the social marketing upstream. The main focus of the upstream approach is on formulating new policies and regulations to make the environment more conducive to the desired behaviour. For many, upstream social marketing admits that individuals should not be wholly responsible for their own behaviour (Dibb, Marylyn Carrigan, \& Gordon, 2013). The upstream approach provides preventive measures that can be implemented by agencies and organisations that will bring about the desired individual behaviour or beyond individual behaviour to influence the behavioural environment at a policy level.

\section{Discussion}

This study sought to extend application of social marketing segmentation in a water use market beyond traditional quantitative methods by investigating whether, using a qualitative technique, focus groups, distinct segments could be identified. Results of the current study facilitated a deep understanding of the target audience. This informed understanding may be used to guide different initiatives to appeal to different types of water users.

The objectives of the current study were twofold. It first aimed to develop a 
context-specific focus group protocol to segment the water use market within one organisational context. The second objective was to understand whether segments could be distinguished using the qualitative research approach. The main finding of the study was that by using a qualitative segmentation approach, distinct segments could be obtained. While there is a growing number of statistical segmentation studies available (Ibrahim, Knox, Rundle-Thiele, \& Arli, 2018; Rundle-Thiele et al., 2015), qualitative segmentation studies within the resource-constrained social marketing field are rare (Randle \& Dolnicar, 2015).

The current study found that comfort segment members shared some characteristics, as was indicated by a U.S. study that segmented lawn irrigators (Warner, Chaudhary, Rumble, Lamm, \& Momol, 2017). Furthermore, comfort users lacked factual knowledge about the UAE's water situation, instead believing that the country has adequate water resources. A social marketing campaign conducted in the U.S. over three years showed that positive messaging and informing people about the true water situation of their environment was able to persuade individuals of their responsibility towards solving, rather than persisting as part of the problem; this campaign reduced water consumption to a more sustainable level (Jesperson, 2005). Askew and McGuirk (2004), in a study conducted in New South Wales, Australia, found that $71 \%$ of the participants were using large quantities of water in their gardens, owing to their lack of knowledge of current water conservation measures. Based on these previous findings, and the unique characteristics of the comfort users' segment within the water use market targeted in this study, disseminating knowledge that presents the true picture of water resources may significantly influence the water consumption behaviours of the majority segment of populations in other locales.

The second segment identified in the current study, classified as careless users, 
consisted mainly of young students living in university accommodation. Their shared common characteristics included excessive water consumption habits, negative attitudes, and inadequate knowledge about the UAE's water resources. Segments similar to the careless users have emerged in other studies examining an audience segmentation approach to water resources, such as the study of home landscape irrigation conducted in the U.S. (Warner et al., 2017).

A third segment emerging from this study, the price- sensitive users were, in the main, older and educated participants who lived in university housing, and enjoyed free water. Their preparedness to change their, and their families' consumption habits was found to depend on the cost of water to themselves. The literature affirms that the water consumption behaviour of a target audience can be influenced by structural change methods such as pricing and/or the imposition of water restrictions. For example, the study of 2179 participants conducted by Randolph and Troy (2008) in the Australian Capital Territory, which used a mixed method approach to researching water use behaviours through focus groups and telephone surveys, identified that tougher water restrictions and increased prices moderated demand.

The fourth, and most demographically diverse segment to emerge from the current study was called the contradictory users' segment. Although Islamic belief did not feature in the focus group protocol, in each of the focus group sessions, at least one participant introduced the importance of religious guidelines relevant to the use of water resources. The ensuing discussion indicated that some participants perceived a misalignment between their faith and their water consumption habits, while others clearly did not. This finding was consistent with a study from the U.S. that, attempting to understand the relationship between self-interest, religion, and the collective good, 
encompassing issues like environmental protection, giving blood, donating to charity, and preferring public transport, revealed conflicts arising between self-interest and concern for the public good (Karp, 1996).

The findings of this study are insightful for social marketing researchers and practitioners whose aim is to reduce water consumption through targeted programs.

This research suggests that different audience segments exist and insights about facilitating factors were identified for each segment. Therefore, tailored social marketing solutions would assist in achieving higher rates of desired behavioural change for each segment.

\section{Facilitating factors}

A number of segment-specific approaches to reducing water consumption are indicated in this study. The excessive consumption habits of careless users could be curbed with technological solutions such as one or more water-efficient appliances (e.g. retrofitted shower heads, timers, and sensors). Comfort users of water could be motivated to change through educational programs, given their lack of accurate knowledge about water scarcity in the UAE. In contrast to the other segments, the contradictory segment could be motivated by appeal to the strength of their religious beliefs. Finally, the price-sensitive segment would only be induced to mitigate their excessive water consumption when a pricing scale applied. The insights gained from segment level analysis point to the range of alternatives that can be implemented to encourage more community members to alter their water use behaviour. The introduction of a price on water would provide a revenue stream to offset education program activities and incentivising installation of fixtures and appliances to support water use reduction. 
478 Because this study was limited to a university-based sample, it is not

479 representative of the whole of UAE society. Participants in this study were relatively highly educated compared to the wider UAE population. Further research is recommended using a broader population base to determine whether the segments that were obtained from this sample and conclusions reached hold for wider UAE 483 contexts. collection such as focus groups, which provided the data for this study. Social desirability bias refers to the tendency of research participants to choose responses they believe are more socially acceptable rather than responses that more accurately reflect their true thoughts or feelings (Grimm, 2010). The dilemma of social

489 desirability bias is that it is most likely to occur in situations such as focus groups, 490 where questions relate to what are widely accepted attitudes, or behavioural or social norms (Grimm, 2010). Alternate research approaches to overcome socially desirable responding are recommended to extend understanding further. Future research that

493 employs different qualitative techniques, such as observation and interviews to 494 consider their utility in segment formation within water use and other contexts is 495 recommended. 


\section{Acknowledgment}

499 The authors would like to acknowledge the participants in this study for their time and

500 effort, and they thank the University of Sharjah for its support in the data collection

501 process.

\section{Declaration of Conflicting Interests}

503 The author(s) declare no potential conflicts of interest with respect to the research,

504 authorship, and/or publication of this article.

\section{Funding}

506 The author(s) received financial support from Griffith University to enable the

507 research, authorship, and/or publication of this article.

508

509

510

511

512

513

514

515

516

517

518

519

520

521

522 


\section{References:}

Andreasen. (2002). Marketing social marketing in the social change marketplace. Journal of Public Policy \& Marketing, 21(1), 3-13. doi: DOI 10.1509/jppm.21.1.3.17602

Andreasen. (2012). Rethinking the relationship between social/nonprofit marketing and commercial marketing. Journal of Public Policy \& Marketing, 31(1), 3641.

Askew, L., E, \& McGuirk, P. M. (2004). Watering the suburbs: distinction, conformity and the suburban garden. Australian Geographer, 35(1), 17-37.

Atkinson, P., \& Hammersley, M. (1994). Ethnography and participant observation.

Balci, G., \& Cetin, I. B. (2017). Market segmentation in container shipping services: A qualitative study. Management Research Review(just-accepted), 00-00.

Berger, Bonime, S. D., Goldberg, L. G., \& White, L. J. (2001). The dynamics of market entry: The effects of mergers and acquisitions on entry in the banking industry.

Berkhin, P. (2006). A survey of clustering data mining techniques. Grouping multidimensional data, 25, 71 .

Bond, J., \& Morris, L. (2003). A class of its own: Latent class segmentation and its implications for qualitative segmentation research. Qualitative Market Research: An International Journal, 6(2), 87-94.

Bryman, A. (2008). Why do researchers integrate/combine $/ \mathrm{mesh} / \mathrm{blend} / \mathrm{mix} / \mathrm{merge} /$ fuse quantitative and qualitative research. Advances in mixed methods research, 87-100.

Carson, D., Gilmore, A., Perry, C., \& Gronhaug, K. (2001). Qualitative marketing research: Sage.

Charmaz, K. (2006). The power of names. Journal of Contemporary Ethnography, 35(4), 396-399.

Creswell, J. W. (2012). Qualitative inquiry and research design: Choosing among five approaches: Sage.

de Visser, R. O., Graber, R., Hart, A., Abraham, C., Scanlon, T., Watten, P., \& Memon, A. (2015). Using qualitative methods within a mixed-methods approach to developing and evaluating interventions to address harmful alcohol use among young people. Health Psychology, 34(4), 349.

Dibb. (2017). Changing Times for Social Marketing Segmentation In T. Dietrich, Sharyn Rundle-Thiele, \& Krzysztof Kubacki (Ed.), Segmnetation in Social Marketing (Vol. 1, pp. 41-60). Singapore: Springer

Dibb, \& Carrigan, M. (2013). Social marketing transformed. European Journal of Marketing, 47(9), 1376-1398. doi: http://dx.doi.org/10.1108/EJM-05-20130248

Dibb, Marylyn Carrigan, S., \& Gordon, R. (2013). Unlocking the potential of upstream social marketing. European Journal of Marketing, 47(9), 1525-1547.

Dietrich, T., Rundle-Thiele, S., \& Kubacki, K. (2017). Segmentation in Social Marketing (Vol. 1). Singapore.

Dolnicar, S., Grün, B., \& Leisch, F. (2016). Increasing sample size compensates for data problems in segmentation studies. Journal of Business Research, 69(2), 992-999.

Donovan, \& Henley, N. (2003). Social marketing: Principles and practice: IP communications Melbourne. 
Esposito, N. (2001). From meaning to meaning: the influence of translation techniques on non-English focus group research. Qualitative Health Research, 11(4), 568-579.

French. (2017). The Importance of Segmentation in Social Marketing Strategy Segmentation in Social Marketing (pp. 25-40): Springer.

French, \& Gordon. (2015). Strategic social marketing: SAGE.

Garee, \& Schori, T. (1996). Focus groups illuminate quantitative research. Marketing News, 30(20), 41-43.

Ghaith, F. A., \& Abusitta, R. (2014). Energy analyses of an integrated solar powered heating and cooling systems in UAE. Energy and Buildings, 70, 117-126.

Glik, D., Prelip, M., Myerson, A., \& Eilers, K. (2008). Fetal alcohol syndrome prevention using community-based narrowcasting campaigns. Health Promotion Practice, 9(1), 93-103.

Grier, S., \& Bryant, C. A. (2005). Social marketing in public health. Annu. Rev. Public Health, 26, 319-339.

Grimm, P. (2010). Social desirability bias. Wiley international encyclopedia of marketing.

Grougiou, V., \& Pettigrew, S. (2009). Seniors' attitudes to voicing complaints: a qualitative study. Journal of Marketing Management, 25(9-10), 987-1001.

Guba, E. G., \& Lincoln, Y. S. (1994). Competing paradigms in qualitative research. Handbook of qualitative research, 2(163-194), 105.

Haque, M., Al-Khaiat, H., \& John, B. (2007). Climatic zones-A prelude to designing durable concrete structures in the Arabian Gulf. Building and environment, 42(6), 2410-2416.

Hofmeister-Tóth, Á., Kelemen, K., \& Piskóti, M. (2011). Environmentally conscious consumption patterns in Hungarian households. Society and Economy, 33(1), 51-68.

Huhman, M., Bauman, A., \& Bowles, H. R. (2008). Initial outcomes of the VERB ${ }^{\text {TM }}$ campaign: tweens' awareness and understanding of campaign messages. American journal of preventive medicine, 34(6), S241-S248.

Ibrahim, A., Knox, K., Rundle-Thiele, S., \& Arli, D. (2018). Segmenting a Water Use Market: Theory of Interpersonal Behavior Insights. Social Marketing Quarterly, 24(1), 3-17.

Jain, A. K. (2010). Data clustering: 50 years beyond K-means. Pattern recognition letters, 31(8), 651-666.

Jesperson, K. (2005). There's an easier way to save water-accentuate the positive: How social marketing makes a difference. On Tap, 19-23.

Kamada, M., Kitayuguchi, J., Inoue, S., Ishikawa, Y., Nishiuchi, H., Okada, S., . . . Shiwaku, K. (2013). A community-wide campaign to promote physical activity in middle-aged and elderly people: a cluster randomized controlled trial. International Journal of Behavioral Nutrition and Physical Activity, 10(1), 44.

Karp, D. G. (1996). Values and their effect on pro-environmental behavior. Environment and behavior, 28(1), 111-133.

Keihner, A. J., Meigs, R., Sugerman, S., Backman, D., Garbolino, T., \& Mitchell, P. (2011). The Power Play! Campaign's School Idea \& Resource Kits improve determinants of fruit and vegetable intake and physical activity among fourthand fifth-grade children. Journal of nutrition education and behavior, 43(4), S122-S129. 
Krueger, R. A., \& Casey, M. A. (2014). Focus groups: A practical guide for applied research: Sage publications.

Kubacki, K., \& Rundle-Thiele, S. (2017). Formative Research in Social Marketing.

Kubacki, K., Rundle-Thiele, S., Pang, B., \& Buyucek, N. (2015). Minimizing alcohol harm: A systematic social marketing review (2000-2014). Journal of Business Research, 68(10), 2214-2222.

Lefebvre, R. C. (2011). An integrative model for social marketing. Journal of Social Marketing, 1(1), 54-72.

Lefebvre, R. C. (2013). Social marketing and social change: Strategies and tools for improving health, well-being, and the environment: John Wiley \& Sons.

Longfield, K., Moorsmith, R., Peterson, K., Fortin, I., Ayers, J., \& Lupu, O. (2016). Qualitative Research for Social Marketing: One Organization's Journey to Improved Consumer Insight. The Qualitative Report, 21(1), 71-86.

Lowe, B., Lynch, D., \& Lowe, J. (2014). The role and application of social marketing in managing water consumption: a case study. International Journal of Nonprofit and Voluntary Sector Marketing, 19(1), 14-26.

Mekonnen, M. M., \& Hoekstra, A. Y. (2016). Four billion people facing severe water scarcity. Science advances, 2(2), e1500323.

Mohamed, M. M., \& Al-Mualla, A. A. (2010). Water demand forecasting in Umm AlQuwain (UAE) using the IWR-MAIN specify forecasting model. Water resources management, 24(14), 4093-4120.

Murad, A. A., Al Nuaimi, H., \& Al Hammadi, M. (2007). Comprehensive assessment of water resources in the United Arab Emirates (UAE). Water Resources Management, 21(9), 1449-1463.

Patton, M. Q. (2005). Qualitative research: Wiley Online Library.

Purdy, C. H. (2011). Using the Internet and social media to promote condom use in Turkey. Reproductive health matters, 19(37), 157-165.

Randle, M., \& Dolnicar, S. (2015). The characteristics of potential environmental volunteers: implications for marketing communications. Australasian Journal of Environmental Management, 22(3), 329-339.

Randle, M., \& Dolnicar, S. (2017). Increasing Civic Engagement Through Market Segmentation Segmentation in Social Marketing (pp. 129-142): Springer.

Randolph, B., \& Troy, P. (2008). Attitudes to conservation and water consumption. environmental science \& policy, 11(5), 441-455.

Reporter. (2016, 22/3/2016). 550 litres of water used per day by a UAE resident Khaleej Times.

Rundle-Thiele, Kubacki, K., Tkaczynski, A., \& Parkinson, J. (2015). Using two-step cluster analysis to identify homogeneous physical activity groups. Marketing Intelligence \& Planning, 33(4), 522-537.

Sandelowski, M. (1995). Sample size in qualitative research. Research in nursing \& health, 18(2), 179-183.

Stokes, D., \& Bergin, R. (2006). Methodology or "methodolatry"? An evaluation of focus groups and depth interviews. Qualitative Market Research: An International Journal, 9(1), 26-37.

Szabo. (2011). The Water Challenge in the UAE. Retrieved from http://cpc.geodata.soton.ac.uk/resources/downloads/dsgpb29eng.pdf website:

Warner, L. A., Chaudhary, A. K., Rumble, J. N., Lamm, A. J., \& Momol, E. (2017). Using Audience Segmentation to Tailor Residential Irrigation Water Conservation Programs. Editing Managing Board, 58(1), 313-333. 
669 Wedel, M., \& Kamakura, W. A. (2012). Market segmentation: Conceptual and

670 methodological foundations (Vol. 8): Springer Science \& Business Media.

671 Weinstein, A., \& Cahill, D. J. (2014). Lifestyle market segmentation: Routledge.

672 Yin, R. K. (2012). Case study methods.

673 Zikmund, McLeod, R., \& Gilbert, F. W. (2003). Customer relationship management:

$674 \quad$ Integrating marketing strategy and information technology: Wiley New York, $675 \quad$ NY.

676 